\title{
Measurement of mixing and CP violation parameters in two-body charm decays
}

\section{The LHCb Collaboration LHC}

Abstract: A study of mixing and indirect $\mathrm{CP}$ violation in $D^{0}$ mesons through the determination of the parameters $y_{\mathrm{CP}}$ and $A_{\Gamma}$ is presented. The parameter $y_{\mathrm{CP}}$ is the deviation from unity of the ratio of effective lifetimes measured in $D^{0}$ decays to the CP eigenstate $K^{+} K^{-}$with respect to decays to the Cabibbo favoured mode $K^{-} \pi^{+}$. The result measured using data collected by LHCb in 2010, corresponding to an integrated luminosity of $29 \mathrm{pb}^{-1}$, is $y_{\mathrm{CP}}=\left(5.5 \pm 6.3_{\text {stat }} \pm 4.1_{\text {syst }}\right) \times 10^{-3}$. The parameter $A_{\Gamma}$ is the asymmetry of effective lifetimes measured in decays of $D^{0}$ and $\bar{D}^{0}$ mesons to $K^{+} K^{-}$. The result is $A_{\Gamma}=\left(-5.9 \pm 5.9_{\text {stat }} \pm 2.1_{\text {syst }}\right) \times 10^{-3}$. A data-driven technique is used to correct for lifetime-biasing effects.

KEYwORDS: Hadron-Hadron Scattering

ArXiv EPRINT: 1112.4698 


\section{Contents}

1 Introduction $\quad 1$

2 Data selection $\quad 3$

2.1 Trigger selection 3

2.2 Offline selection 4

3 Determination of decay-time acceptance effects 4

$\begin{array}{lll}4 & \text { Fitting method } & 6\end{array}$

5 Cross-checks and systematic uncertainties $\quad 9$

$\begin{array}{ll}5.1 \text { Evaluation of systematic uncertainties } & 10\end{array}$

$\begin{array}{ll}5.2 \text { Summary of systematic uncertainties } & 11\end{array}$

$6 \quad$ Results and conclusion $\quad 12$

$\begin{array}{lr}\text { The LHCb collaboration } & 16\end{array}$

\section{Introduction}

Mixing of neutral $D^{0}$ mesons has only recently been established [1-3] and first evidence for $\mathrm{CP}$ violation in the charm sector has just been observed by LHCb [4]. In this work the mixing and $\mathrm{CP}$ violation parameters $y_{\mathrm{CP}}$ and $A_{\Gamma}$ in the decays of neutral $D^{0}$ mesons into two charged hadrons are studied. Both quantities are measured here for the first time at a hadron collider. The observable $y_{\mathrm{CP}}$ is the deviation from unity of the ratio of effective lifetimes in the decay modes $D^{0} \rightarrow K^{-} \pi^{+}$and $D^{0} \rightarrow K^{-} K^{+}$

$$
y_{\mathrm{CP}} \equiv \frac{\tau\left(D^{0} \rightarrow K^{-} \pi^{+}\right)}{\tau\left(D^{0} \rightarrow K^{-} K^{+}\right)}-1
$$

where the effective lifetime is defined as the value measured using a single exponential model. All decays implicitly include their charge conjugate modes, unless explicitly stated otherwise. Similarly, $A_{\Gamma}$ is given by the asymmetry of effective lifetimes as

$$
A_{\Gamma} \equiv \frac{\tau\left(\bar{D}^{0} \rightarrow K^{+} K^{-}\right)-\tau\left(D^{0} \rightarrow K^{+} K^{-}\right)}{\tau\left(\bar{D}^{0} \rightarrow K^{+} K^{-}\right)+\tau\left(D^{0} \rightarrow K^{+} K^{-}\right)} .
$$

The neutral $D^{0}$ mass eigenstates $\left|D_{1,2}\right\rangle$ with masses $m_{1,2}$ and widths $\Gamma_{1,2}$ can be expressed as linear combinations of the flavour eigenstates as $\left|D_{1,2}\right\rangle=p\left|D^{0}\right\rangle \pm q\left|\bar{D}^{0}\right\rangle$ with complex coefficients $p$ and $q$ satisfying $|p|^{2}+|q|^{2}=1$. The average mass and width are defined as $m \equiv\left(m_{1}+m_{2}\right) / 2$ and $\Gamma \equiv\left(\Gamma_{1}+\Gamma_{2}\right) / 2$; the mass and width difference are 
used to define the mixing parameters $x \equiv\left(m_{2}-m_{1}\right) / \Gamma$ and $y \equiv\left(\Gamma_{2}-\Gamma_{1}\right) /(2 \Gamma)$. The phase convention is chosen such that $\mathrm{CP}\left|\mathrm{D}^{0}\right\rangle=-\left|\overline{\mathrm{D}}^{0}\right\rangle$ and $\mathrm{CP}\left|\overline{\mathrm{D}}^{0}\right\rangle=-\left|\mathrm{D}^{0}\right\rangle$ which leads, in the case of no CP violation $(p=q)$, to $\left|D_{1}\right\rangle$ being the CP odd and $\left|D_{2}\right\rangle$ the CP even eigenstate, respectively.

The parameter

$$
\lambda_{f}=\frac{q \bar{A}_{f}}{p A_{f}}=-\eta_{\mathrm{CP}}\left|\frac{q}{p}\right|\left|\frac{\bar{A}_{f}}{A_{f}}\right| e^{i \phi},
$$

contains the amplitude $A_{f}\left(\bar{A}_{f}\right)$ of $D^{0}\left(\bar{D}^{0}\right)$ decays to the CP eigenstate $f$ with eigenvalue $\eta_{\mathrm{CP}}$. The mixing parameters $x$ and $y$ are known to be at the level of $10^{-2}$ while both the phase and the deviation of the magnitude from unity of $\lambda_{f}$ are experimentally only constrained to about $0.2[5]$. The direct $\mathrm{CP}$ violation, i.e. the difference in the rates of $D^{0}$ and $\bar{D}^{0}$ decays, is constrained to the level of $10^{-2}$ and has recently been measured by LHCb [4]. Introducing $|q / p|^{ \pm 2} \approx 1 \pm \mathrm{A}_{\mathrm{m}}$ and $\left|\bar{A}_{f} / A_{f}\right|^{ \pm 2} \approx 1 \pm \mathrm{A}_{\mathrm{d}}$, with the assumption that $\mathrm{A}_{\mathrm{m}}$ and $\mathrm{A}_{\mathrm{d}}$ are small, and neglecting terms below $10^{-4}$ according to the experimental constraints, one obtains according to $[6,7]$

$$
y_{\mathrm{CP}} \approx\left(1-\frac{1}{8} \mathrm{~A}_{\mathrm{m}}^{2}\right) y \cos \phi-\frac{1}{2} \mathrm{~A}_{\mathrm{m}} x \sin \phi .
$$

In the limit of no $\mathrm{CP}$ violation $y_{\mathrm{CP}}$ is equal to $y$ and hence becomes a pure mixing parameter. However, once precise measurements of $y$ and $y_{\mathrm{CP}}$ are available, any difference between $y$ and $y_{\mathrm{CP}}$ would be a sign of $\mathrm{CP}$ violation.

Previous measurements of $y_{\mathrm{CP}}$ have been performed by BaBar and Belle. The results are $y_{\mathrm{CP}}=(11.6 \pm 2.2 \pm 1.8) \times 10^{-3}[8]$ for BaBar and $y_{\mathrm{CP}}=(13.1 \pm 3.2 \pm 2.5) \times 10^{-3}[2]$ for Belle. They are consistent with the world average of $y=(7.5 \pm 1.2) \times 10^{-3}[5]$.

The study of the lifetime asymmetry of $D^{0}$ and $\bar{D}^{0}$ mesons decaying into the singly Cabibbo-suppressed final state $K^{+} K^{-}$can reveal indirect CP violation in the charm sector. The measurement can be expressed in terms of the quantity $A_{\Gamma}$. Using the same expansion as for $y_{\mathrm{CP}}$ leads to

$$
\begin{aligned}
A_{\Gamma} & \approx\left[\frac{1}{2}\left(\mathrm{~A}_{\mathrm{m}}+\mathrm{A}_{\mathrm{d}}\right) y \cos \phi-x \sin \phi\right] \frac{1}{1+y_{\mathrm{CP}}} \\
& \approx \frac{1}{2}\left(\mathrm{~A}_{\mathrm{m}}+\mathrm{A}_{\mathrm{d}}\right) y \cos \phi-x \sin \phi .
\end{aligned}
$$

Despite this measurement being described in most literature as a determination of indirect $\mathrm{CP}$ violation by neglecting the term proportional to $\mathrm{A}_{\mathrm{d}}$, it is apparent that direct $\mathrm{CP}$ violation at the level of $10^{-2}$ can have a contribution to $A_{\Gamma}$ at the level of $10^{-4}$. Therefore precise measurements of both time-dependent and time-integrated asymmetries are necessary to reveal the nature of $\mathrm{CP}$ violating effects in the $D^{0}$ system.

The measurement of $A_{\Gamma}$ requires tagging the flavour of the $D^{0}$ at production, which will be discussed in the following section. Previous measurements of $A_{\Gamma}$ were performed by Belle and BaBar leading to $A_{\Gamma}=(0.1 \pm 3.0 \pm 1.5) \times 10^{-3}[2]$ and $A_{\Gamma}=(2.6 \pm 3.6 \pm 0.8) \times 10^{-3}[9]$, respectively. They are consistent with zero, hence showing no indication of $\mathrm{CP}$ violation. 


\section{Data selection}

$\mathrm{LHCb}$ is a precision heavy flavour experiment which exploits the abundance of charm particles produced in collisions at the Large Hadron Collider (LHC). The LHCb detector [10] is a single arm spectrometer at the LHC with a pseudorapidity acceptance of $2<\eta<5$ for charged particles. High precision measurements of flight distances are provided by the Vertex Locator (VELO), which consists of two halves with a series of semi-circular silicon microstrip detectors. The VELO measurements, together with momentum information from forward tracking stations and a $4 \mathrm{Tm}$ dipole magnet, lead to decay-time resolutions of the order of one tenth of the $D^{0}$ lifetime. Two Ring-Imaging Cherenkov (RICH) detectors using three different radiators provide excellent pion-kaon separation over the full momentum range of interest. The detector is completed by hadronic and electromagnetic calorimeters and muon stations. The measurements presented here are based on a data sample corresponding to an integrated luminosity of $29 \mathrm{pb}^{-1}$ of $p p$ collisions at $\sqrt{s}=7 \mathrm{TeV}$ recorded during the LHC run in 2010.

\subsection{Trigger selection}

The LHCb trigger consists of hardware and software (HLT) stages. The hardware trigger is responsible for reducing the LHC $p p$ interaction rate from $\mathcal{O}(10) \mathrm{MHz}$ to the rate at which the LHCb subdetectors can be read out, nominally $1 \mathrm{MHz}$. It selects events based on the transverse momentum of track segments in the muon stations, the transverse energy of clusters in the calorimeters, and overall event multiplicity.

The HLT further reduced the event rate to about $2 \mathrm{kHz}$ in 2010, at which the data was stored for offline processing. The HLT runs the same software for the track reconstruction and event selection as is used offline and has access to the full event information.

The first part of the HLT is based on the reconstruction of tracks and primary interaction vertices in the VELO. Heavy flavour decays are identified by their large lifetimes, which cause their daughter tracks to be displaced from the primary interaction. The trigger first selects VELO tracks whose distance of closest approach to any primary interaction, known as the impact parameter (IP), exceeds $110 \mu \mathrm{m}$. In addition the tracks are required to have at least ten hits in the VELO to reduce further the accepted rate of events. This cut limits the fiducial volume for $D^{0}$ decays and therefore rejects events where the $D^{0}$ candidate has a large transverse component of the distance of flight, causing an upper bound on the decay-time acceptance. The term decay-time acceptance will be used throughout this paper to refer to the selection efficiency as a function of the $D^{0}$ decay time. Selected tracks are then used to define a region of interest in the tracking stations after the dipole magnet, whose size is defined by an assumed minimum track momentum of $8 \mathrm{GeV} / c$; hits inside these search regions are used to form tracks traversing the full tracking system. Tracks passing this selection are fitted, yielding a full covariance matrix, and a final selection is made based on the track-fit quality and the track $\chi^{2}(\mathrm{IP})$. The $\chi^{2}(\mathrm{IP})$ is a measure of the consistency with the hypothesis that the IP is equal to zero. At least one good track is required for the event to be accepted. The requirements on both the track IP and on the $\chi^{2}(\mathrm{IP})$ reduce the number of $D^{0}$ candidates with a short decay time. 
In the second part of the HLT, an exclusive selection of $D^{0}$ candidates is performed by reconstructing two-track vertices. Further cuts are placed on the $\chi^{2}$ (IP) of the $D^{0}$ daughters and the displacement significance of the $D^{0}$ vertex from the primary interaction, as well as a requirement which limits the collinearity angle between the $D^{0}$ momentum and the direction of flight, as defined by the primary and decay vertices. These cuts all affect the distribution of the decay time of the $D^{0}$ candidates. Additional cuts are placed on track and vertex fit quality, and on kinematic quantities such as the transverse momentum of the $D^{0}$ candidate, which have no effect on the decay-time distribution.

\subsection{Offline selection}

Given the abundance of charm decays, the selection has been designed to achieve high purity. It uses similar requirements to those made in the trigger selection, though often with tighter thresholds. In addition it makes use of the RICH information for separating kaons and pions. A single kaon is positively identified with an efficiency of on average about $83 \%$, while less than $5 \%$ of the pions are wrongly identified as kaons, when taking into account the momentum distribution of the decay products. A mass window of $\pm 16 \mathrm{MeV} / \mathrm{c}^{2}$ (about $\pm 2 \sigma$ ) is applied to the invariant mass of the two $D^{0}$ daughter particles using the appropriate mass hypotheses. After these criteria have been applied there is negligible remaining cross-feed between the different two-body $D^{0}$ decay modes.

Flavour tagging of the $D^{0}$ decays is done by reconstructing the $D^{*+} \rightarrow D^{0} \pi_{\mathrm{s}}^{+}$decay, where the charge of the slow pion, $\pi_{\mathrm{s}}$, determines the flavour of the $D^{0}$ meson at production. The selection applies loose requirements on the kinematics of the bachelor pion and the quality of the $D^{*+}$ vertex fit. The most powerful variable for selecting the $D^{*+}$ decay is the difference in the reconstructed invariant masses of the $D^{*+}$ and the $D^{0}$ candidates, $\Delta m$. Candidates are required to have $\Delta m$ in the range $\left|\Delta m-145.4 \mathrm{MeV} / c^{2}\right|<2.0 \mathrm{MeV} / c^{2}$.

Events with multiple signal candidates are excluded from the analysis. For tagged $D^{0}$ decays this causes a reduction of the number of candidates of about $15 \%$ due to the high probability of assigning a random slow pion to form a $D^{*+}$ candidate. The numbers of selected candidates are 286155 for $D^{0} \rightarrow K^{-} \pi^{+}$and 39262 for $D^{0} \rightarrow K^{+} K^{-}$decays.

\section{Determination of decay-time acceptance effects}

Since absolute lifetime measurements are used to extract $y_{\mathrm{CP}}$ and $A_{\Gamma}$, it is essential to correct for lifetime-biasing effects. The analysis uses a data-driven approach that calculates, for each candidate and at every possible decay time, an acceptance value of zero or one which is related to the trigger decision and offline selection. The method used to determine decay-time acceptance effects is based on the so-called "swimming" algorithm. This approach was first used at the NA11 spectrometer [11], further developed within DELPHI [12] and CDF [13, 14], studied at LHCb [15, 16], and applied to the measurement of the $B_{s}^{0} \rightarrow K^{+} K^{-}$lifetime [17].

Lifetime-biasing effects originate from selection criteria or from efficiencies that depend on the decay time. The swimming method accounts for selection biases. Efficiency effects are estimated and, where necessary, corrected for as described at the end of this section. 
The swimming method relies on the fact that the selection criteria which can cause a bias depend on the geometry of the specific decay, while the probability of a decay to occur with this geometry is independent of the decay time.

The per-event acceptance at any given decay time can be 1 to signify that the event would have been triggered or selected at that decay time, or 0 to show that it would have been rejected. The values are 0 or 1 as the overall selection efficiency factorises out. One example of a requirement that causes a non-trivial decay time acceptance is that on the minimum value of the impact parameter of the decay product tracks. An impact parameter is the closest distance of approach of an extrapolated track to the primary interaction vertex. Such a selection criterion leads to a step in the acceptance as a function of decay-time as shown in figure 1. The acceptance is, of course, 1 at the measured decay time of that event, $t_{\text {meas }}$. However, the location of the step in the function depends on the geometry of the event and does not depend on $t_{\text {meas }}$.

Several effects can lead to a more complex shape of the acceptance function than a single step. A second primary interaction vertex can for example lead to a gap in the acceptance for the decay-time range, for which the impact parameter of one track with respect to this second vertex falls below the threshold. Therefore, the general per-event acceptance function can be described by a series of steps, called "turning points".

The acceptance function is used in the normalisation of the decay-time probability density function (PDF). The single-event probability density of measuring a decay at time $t$, ignoring measurement errors, is given by

$$
f_{\mathrm{t}}(t \mid A)=\frac{\frac{1}{\tau} e^{-t / \tau} \Theta(t) A(t)}{\int_{-\infty}^{\infty} \frac{1}{\tau} e^{-t^{\prime} / \tau} \Theta\left(t^{\prime}\right) A\left(t^{\prime}\right) d t^{\prime}},
$$

where $\tau$ is the average lifetime of the decay, $\Theta(t)$ is the Heaviside function, and $A(t)$ is the decay-time acceptance function for this candidate. If the event-by-event acceptance function, $A(t)$, consists of pairs of turning points, $\left(t_{\min , \mathrm{i}}, t_{\mathrm{max}, \mathrm{i}}\right)$, eq. (3.1) leads to

$$
f_{\mathrm{t}}(t \mid A)=\frac{\frac{1}{\tau} e^{-t / \tau} \Theta(t)}{\sum_{i}\left[e^{-t_{\max , \mathrm{i}} / \tau}-e^{-t_{\min , \mathrm{i}} / \tau}\right]},
$$

with $i$ summing over the pairs of acceptance turning points and assuming that $t$ lies in an accepted time interval, i.e. $t_{\min , \mathrm{i}}<t<t_{\max , \mathrm{i}}$ or $A(t)=1$, and $f_{\mathrm{t}}(t \mid A)=0$ if $A(t)=0$.

The swimming method determines the turning points of the per-event acceptance by moving the primary interaction vertex in steps along the direction of the $D^{0}$ momentum. At each step the selection decision is evaluated which yields the value of the acceptance function corresponding to the decay time of this step. The decay time is calculated using the distance of the moved primary interaction vertex to the decay vertex. In events containing multiple primary vertices, all are moved by the same amount in the direction of the $D^{0}$ momentum. This procedure is executed twice: once for the trigger selection and once for the offline selection. The two resulting acceptance functions are combined to a single acceptance function by including only the ranges which have been accepted by both steps.

The novelty in this implementation of the swimming method is the ability to execute the $\mathrm{LHCb}$ trigger, including the reconstruction, in precisely the same configuration used 


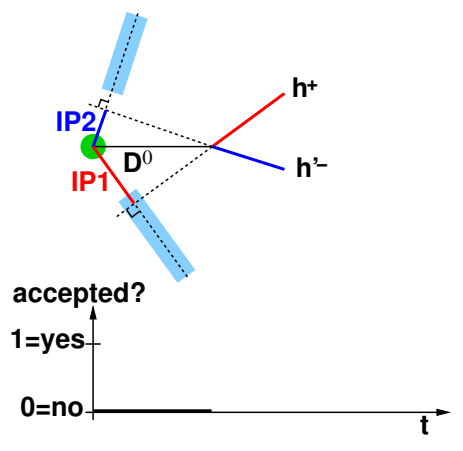

(a)

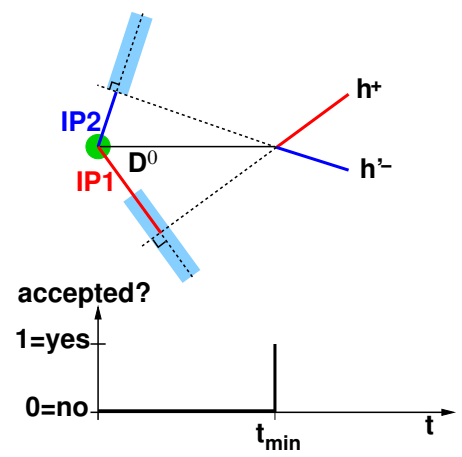

(b)

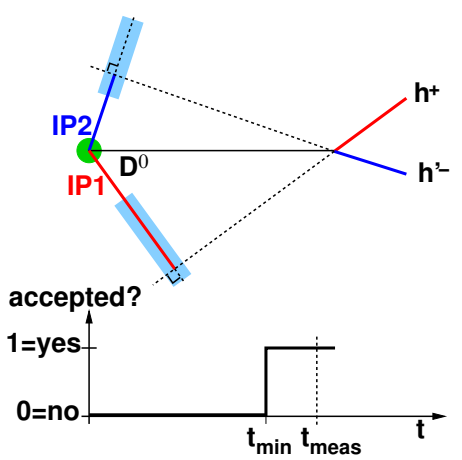

(c)

Figure 1. Evolution of the decay-time acceptance function for a two-body $D^{0}$ decay. The shaded, light blue regions show the bands for accepting a track impact parameter. While the impact parameter of the negative track (IP2) is too low in (a) it reaches the accepted range in (b). The actual measured decay time, $t_{\text {meas }}$, lies in the accepted region which continues to larger decay times (c).

during data taking. This is made possible by the implementation of all lifetime-biasing trigger requirements being in software as opposed to hardware.

Studying the decay-time dependence of the acceptance in principle requires moving the hits produced by the $D^{0}$ decay products, rather than the primary vertices. Our implementation leads to significant technical simplifications. This ignores the fact that events are no longer accepted if the mother particle has such a long decay time that one or both tracks can no longer be reconstructed inside the VELO. This is a very small effect as a $D^{0}$ meson has to fly ten to a hundred times its average distance of flight in order to escape detection in the VELO. Nevertheless, this effect has been estimated based on the knowledge of the position of the VELO modules and on the number of hits required to form a track. The limit of the acceptance is determined by swimming the tracks along the $D^{0}$ momentum vector. The result is treated as another per event decay-time acceptance and merged with the acceptance of the trigger and offline selections.

Finally, the track reconstruction efficiency in the trigger is reduced compared to the offline reconstruction due to the requirements described in section 2. It has been verified, using a smaller sample acquired without a lifetime biasing selection, that this relative reconstruction efficiency does not depend on the decay time of the $D^{0}$ candidate with a precision of $3 \times 10^{-3}$, and therefore introduces no significant additional acceptance effect.

\section{Fitting method}

The peak in $\Delta m$ from true $D^{*+}$ decays is parametrised as the sum of three Gaussians; two of which have a common mean and a third which has a slightly higher mean. The random $\pi_{\mathrm{s}}$ background PDF is given by

$$
f_{\pi_{\mathrm{s}}}(\Delta m)=\left(\frac{\Delta m}{a}\right)^{2}\left(1-\exp \left(-\frac{\Delta m-d}{c}\right)\right)+b\left(\frac{\Delta m}{d}-1\right) \quad \Delta m \geq d
$$




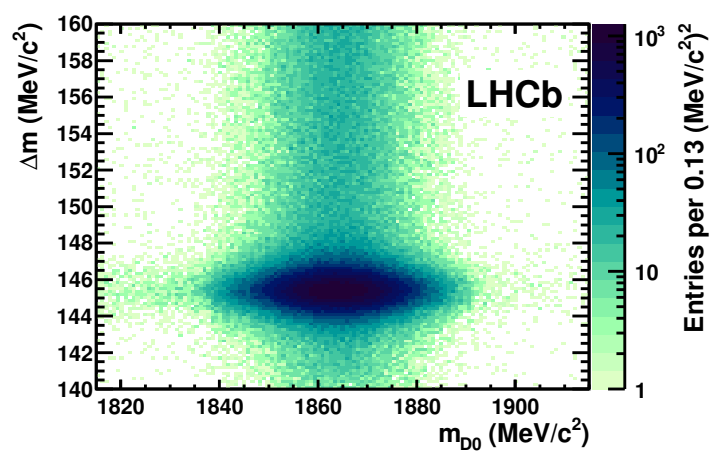

Figure 2. $\Delta m$ vs $m_{D^{0}}$ distribution for $D^{0} \rightarrow K^{-} \pi^{+}$candidates. The contribution of random slow pions extends around the signal peak in the vertical direction while background is visible as a horizontal band.
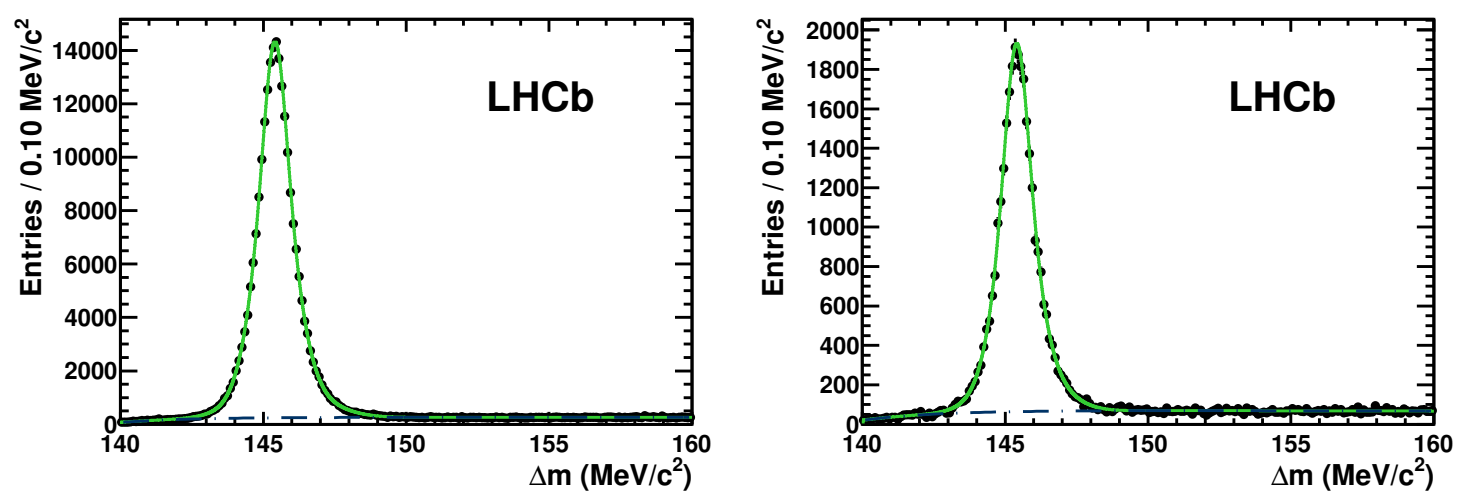

Figure 3. $\Delta m$ fit projections of (left) $D^{0} \rightarrow K^{-} \pi^{+}$and (right) $D^{0} \rightarrow K^{+} K^{-}$candidates to which the full offline selection apart from the cut in $\Delta m$ has been applied. Shown are data (points), the total fit (green, solid) and the background component (blue, dot-dashed).

where $a$ and $b$ define the slope at high values of $\Delta m, c$ defines the curvature at low values of $\Delta m$ and $\Delta m=d$ defines the threshold below which the function is equal to zero. Figure 2 shows the $\Delta m$ vs $m_{D^{0}}$ distribution and figure 3 shows the fit to the mass difference between the reconstructed invariant masses of $D^{*+}$ and $D^{0}$ candidates, $\Delta m$.

The signal yield is extracted from fits to the reconstructed $D^{0}$ invariant mass distribution after application of the cut in $\Delta m$. The fit model for the signal peak has been chosen to be a double Gaussian and background is modelled as a first-order polynomial. The background level is evaluated to be about $1 \%$ for $D^{0} \rightarrow K^{-} \pi^{+}$decays and about $3 \%$ for $D^{0} \rightarrow K^{+} K^{-}$decays. It consists of combinatorial background and partially reconstructed or misidentified $D^{0}$ decays. If the latter stem from a $D^{*+}$ decay they have a peaking distribution in $\Delta m$ similar to signal candidates. The data in the mass sidebands are insufficient to reliably describe the background shape in other variables, so the background contribution is neglected in the time-dependent fit and a systematic uncertainty is estimated accordingly. All fits are carried out as unbinned maximum likelihood fits. 
Events inside the signal windows in $\Delta m$ and $m_{D^{0}}$ are used in the lifetime fit, where $D^{0}$ mesons produced at the primary vertex (prompt) have to be distinguished from those originating from $b$ hadron decays (secondary). The combined PDF for this decay-time dependent fit is factorized as

$$
f\left(\chi^{2}\left(\mathrm{IP}_{D}\right), t, A\right)=\sum_{\substack{\text { class } \\ \text { =prompt, } \\ \text { secondary }}} f_{\mathrm{IP}}\left(\chi^{2}\left(\mathrm{IP}_{D}\right) \mid t, A, \text { class }\right) f_{\mathrm{t}}(t \mid A, \text { class }) f_{\mathrm{TP}}(A \mid \text { class }) P(\text { class }) .
$$

The four factors on the right-hand side of eq. (4.2), which will be described in detail below, are:

- the time-dependent PDFs for the $\ln \chi^{2}\left(\operatorname{IP}_{D}\right)$ values for prompt and secondary $D^{0}$ mesons;

- the decay-time PDFs for prompt and secondary $D^{0}$ mesons;

- the PDF for the turning points which define the acceptance $A$;

- the fractions of prompt and secondary $D^{0}$ decays among the signal candidates.

The separation of prompt and secondary $D^{0}$ mesons is done on a statistical basis using the impact parameter of the $D^{0}$ candidate with respect to the primary vertex, $\operatorname{IP}_{D}$. For prompt decays, this is zero up to resolution effects, but can acquire larger values for secondary decays as the $D^{0}$ candidate does not in general point back to the primary vertex. Since an estimate of the vertex resolution is available on an event-by-event basis, it is advantageous to use the $\chi^{2}$ of the $\mathrm{IP}_{D}$ instead of the impact parameter value itself. The natural logarithm of this quantity, $\ln \left(\chi^{2}\left(\mathrm{IP}_{D}\right)\right)$, allows for an easier parametrisation. Empirically, the sum of two bifurcated Gaussians, i.e. Gaussians with different widths on each side of the mean, and a third, symmetric Gaussian, all sharing a common peak position, is found to be a suitable model to describe the $\ln \left(\chi^{2}\left(\operatorname{IP}_{D}\right)\right)$ distribution for both prompt and secondary $D^{0}$ decays.

For the prompt $D^{0}$ class the $\ln \left(\chi^{2}\left(\mathrm{IP}_{D}\right)\right)$ distribution does not change with $D^{0}$ decay time as the true value is zero at all times and the resolution of $\operatorname{IP}_{D}$ can be assumed to be independent of the measured decay time. For secondary $D^{0}$ decays the decay-time and $\ln \left(\chi^{2}\left(\operatorname{IP}_{D}\right)\right)$ are correlated. The width of the $\ln \left(\chi^{2}\left(\operatorname{IP}_{D}\right)\right)$ distribution is found to be approximately constant in decay time for both prompt and secondary $D^{0}$ mesons. As Monte Carlo simulation studies suggest that secondary decays have a larger width in this variable, a scale factor between the widths for prompt and secondary mesons is introduced. The mean value of $\ln \left(\chi^{2}\left(\mathrm{IP}_{D}\right)\right)$ increases with $D^{0}$ decay time, which reflects the fact that $D^{0}$ mesons coming from other long-lived decays do not necessarily point back to the primary vertex and that they may point further away the further their parent particle flies. The functional form for this time dependence is based on simulation and all parameters are determined in the fit to data.

The decay-time PDF, $f_{\mathrm{t}}(t \mid A$, class $)$ is modelled as a single exponential for the prompt $D^{0}$ class and as a convolution of two exponentials for secondary decays. To account for 
resolution effects, these are convolved with a single Gaussian resolution function. The parameters of the resolution model are obtained from a fit to the decay time distribution of prompt $J / \psi$ events. The resulting dilution is equivalent to that of a single Gaussian with a width of $50 \mathrm{fs}$ [18]. The decay-time probability densities are properly normalized by integrating their product with the acceptance function $A$, evaluated by the swimming method, only over the decay-time intervals for which the event would have been accepted. Hence, the acceptance turning points are used as boundaries in the integration.

Finally, a PDF for the per-event acceptance function is needed. While the first acceptance turning point, i.e. the one with the smallest decay time, depends on the $D^{0}$ decay topology, the others are governed more by the underlying event structure, e.g. the distribution of primary vertices. The primary vertex distribution is independent of whether the $D^{0}$ candidate is of prompt or secondary origin. Hence, the PDF can be approximated as $f_{\mathrm{TP}}(A \mid$ class $) \approx f_{\mathrm{TP}}\left(\mathrm{TP}_{1} \mid\right.$ class $)$, where $\mathrm{TP}_{1}$ denotes the position of the first turning point. The distribution for $f_{\mathrm{TP}}\left(\mathrm{TP}_{1} \mid\right.$ prompt $)$ is obtained by applying a cut at $\ln \chi^{2}\left(\mathrm{IP}_{D}\right)<1$, thus selecting a very pure sample of prompt decays. The distribution for $f_{\mathrm{TP}}\left(\mathrm{TP}_{1} \mid\right.$ secondary $)$ is obtained from the distribution of $\mathrm{TP}_{1}$ weighted by the probability of each candidate being of secondary decay origin.

An initial fit is performed using the full $\ln \chi^{2}\left(\mathrm{IP}_{D}\right)$ distribution and all parameters in the description of this term are then fixed in the final fit. A cut is then applied requiring $\ln \chi^{2}\left(\mathrm{IP}_{D}\right)<2$ in order to suppress the fractions of both background and secondary candidates to less than a few percent. The final fit is performed on this reduced sample, which contains $226110 D^{0} \rightarrow K^{-} \pi^{+}$and $30481 D^{0} \rightarrow K^{+} K^{-}$candidates. The effect of this procedure is estimated in the systematic uncertainty evaluation.

\section{Cross-checks and systematic uncertainties}

The method for absolute lifetime measurements described in section 4 comprises three main parts whose accuracy and potential for biasing the measurement have to be evaluated in detail:

- the determination of the event-by-event decay-time acceptance;

- the separation of prompt from secondary charm decays;

- the estimation of the decay time distribution of combinatorial background.

Since the contribution of combinatorial background is ignored in the fit, it is important to evaluate the corresponding systematic uncertainty. Furthermore, several other parameters are used in the fit whose systematic effects have to be evaluated, e.g. the description of the decay-time resolution. It is generally expected that the systematic uncertainties in $y_{\mathrm{CP}}$ are similar to or larger than those in $A_{\Gamma}$ as in $y_{\mathrm{CP}}$ two different final states contribute to the measurement.

Several consistency checks are performed by splitting the dataset into subsets. The stability is tested as a function of run period, $D^{0}$ momentum and transverse momentum, and 
primary vertex multiplicity. No significant trend is observed and therefore no systematic uncertainty assigned.

The fitting procedure is verified using simplified Monte Carlo simulation studies. No indication of a bias is observed and the statistical uncertainties are estimated accurately. A further test is carried out using full Monte Carlo simulation to a relative precision of $0.9 \%$. The acceptance effects are corrected using the same method as applied to data. The generated lifetime is obtained in the fit which implies that the lifetime biasing effects are properly corrected.

As an additional check, a control measurement is performed using the lifetime asymmetry of $D^{0}$ and $\bar{D}^{0}$ decays to the Cabibbo favoured decay $D^{0} \rightarrow K^{-} \pi^{+}$. The result is in agreement with zero and the flavour-averaged $D^{0}$ lifetime is found to be consistent with the world average. Detailed results are given in section 6 . The fit results for $D^{0} \rightarrow K^{+} K^{-}$ decays were not revealed throughout the development of the method and the study of systematic uncertainties for the measurements of $y_{\mathrm{CP}}$ and $A_{\Gamma}$.

\subsection{Evaluation of systematic uncertainties}

Particle decay times are measured from the distance between the primary vertex and secondary decay vertex in the VELO. The systematic uncertainty from the distance scale is determined by considering the potential error on the length scale of the detector from the mechanical survey, thermal expansion and the current alignment precision. A relative systematic uncertainty of $0.1 \%$ is assigned to the measurements of absolute lifetimes, translating into a relative uncertainty of $0.1 \%$ on $A_{\Gamma}$ and $y_{\mathrm{CP}}$.

The method to evaluate the turning points of the decay-time acceptance functions described in section 3 uses an iterative approach which estimates the turning points to a precision of about $1 \mathrm{fs}$. Two scenarios have been tested: a common bias of all acceptance turning points and a common length scaling of the turning points, which could originate from differences in the length scale in the trigger and offline reconstructions. From a variation of the bias and the scale, a systematic uncertainty of $0.1 \times 10^{-3}$ on $A_{\Gamma}$ and $y_{\mathrm{CP}}$ is determined.

The reconstruction acceptance is dominated by the VELO geometry, which is accounted for by the method described in section 3. This leads to a correction of less than 1 fs on the absolute lifetime measurements, i.e. a relative correction of about $0.24 \%$. No further systematic uncertainty is assigned to $A_{\Gamma}$ or $y_{\mathrm{CP}}$ as the size of this relative correction is negligible. Additional studies of the reconstruction efficiency as a function of variables governing the decay geometry did not provide any indication of lifetime biasing effects.

The decay-time resolution is modelled by a single Gaussian. The width of the resolution function is varied from its nominal value of $0.05 \mathrm{ps}$ between $0.03 \mathrm{ps}$ and $0.07 \mathrm{ps}$. The range of variation was chosen to cover possible alignment effects as well as effects from the different final state used to evaluate the resolution. The result leads to a systematic uncertainty of $0.1 \times 10^{-3}$ for $A_{\Gamma}$ and $y_{\mathrm{CP}}$.

The fit range in decay time is restricted by lower and upper limits. The lower limit is put in place to avoid instabilities in regions with extremely low decay-time acceptances and very few events. The default cut value is $0.25 \mathrm{ps}$ which is close to the lower end of the 
observed range of events. This cut is varied to both $0.2 \mathrm{ps}$ and $0.3 \mathrm{ps}$. The result leads to a systematic uncertainty of $0.1 \times 10^{-3}$ for $A_{\Gamma}$ and $0.8 \times 10^{-3}$ for $y_{\mathrm{CP}}$.

The upper limit of the fit range in decay time is put in place to minimise the impact of long-lived background events. The default cut is put at $6 \mathrm{ps}$ which corresponds to about $15 D^{0}$ lifetimes. This cut is varied to $5 \mathrm{ps}$ and $8 \mathrm{ps}$. The result leads to a systematic uncertainty of $0.2 \times 10^{-3}$ for $A_{\Gamma}$ and $y_{\mathrm{CP}}$.

The description of the contribution from combinatorial background is studied by varying its relative amount in the data sample and repeating the fit. This is done by changing the $\Delta m$ window from the default of $\pm 2 \mathrm{MeV} / c^{2}$ to $\pm 1 \mathrm{MeV} / c^{2}$ and $\pm 3 \mathrm{MeV} / c^{2}$. The result leads to a systematic uncertainty of $1.3 \times 10^{-3}$ for $A_{\Gamma}$ and $0.8 \times 10^{-3}$ for $y_{\mathrm{CP}}$.

Events that originate from secondary charm decays are the background with the largest impact on the fit procedure as they have a very different decay-time distribution compared to prompt charm decays, but they peak in the invariant mass and $\Delta m$ distributions. Also a fraction of combinatorial background events appear to be secondary-like in their $\ln \chi^{2}\left(\operatorname{IP}_{D}\right)$ distribution. The cut of $\ln \chi^{2}\left(\operatorname{IP}_{D}\right)<2$ removes a large fraction of secondary-like events. However, it is important that the remainder is properly modelled and does not bias the signal lifetime. Varying this cut changes the relative number of secondary-like decays in the sample and therefore tests the stability of the secondary description in the fit model. The fraction of secondary-like combinatorial background events is also altered with this test. The $\ln \chi^{2}\left(\mathrm{IP}_{D}\right)$ cut is varied from 1.5 which is just above the peak of the prompt distribution to 3.5 where the probability densities for prompt and secondary decays are about equal. The result leads to a systematic uncertainty of $1.6 \times 10^{-3}$ for $A_{\Gamma}$ and $3.9 \times 10^{-3}$ for $y_{\mathrm{CP}}$. The uncertainty is significantly larger for $y_{\mathrm{CP}}$ than for $A_{\Gamma}$ as may be expected from the difference in the background level in the channels involved in the $y_{\mathrm{CP}}$ measurement.

Additional studies were performed to estimate the potential impact of neglecting background events in the fit. A background component was added to a simplified simulation. The background decay time distribution was generated using extreme values of fits to the distribution observed in mass sidebands. The average bias on the measurement of $y_{\mathrm{CP}}$ was about $2 \times 10^{-3}$. Since this is consistent with the assigned systematic uncertainty, we do not assign any additional uncertainty.

Furthermore, a background component was added to the $D^{0}$ decay-time PDF with a fixed fraction and average lifetime. The fraction of this component, which was assumed to be secondary-like, was varied. A change in the fit result for $y_{\mathrm{CP}}$ of 0 (all background secondary-like) to $4 \times 10^{-3}$ (all background prompt-like) was observed. As it is known that a fraction of the background events are secondary-like, this result is considered consistent with the simplified simulation results.

\subsection{Summary of systematic uncertainties}

Table 1 summarises the systematic uncertainties evaluated as described above. The main systematic uncertainties are due to neglecting the combinatorial background and to the contribution of secondary-like decays. The total systematic uncertainties for $A_{\Gamma}$ and $y_{\mathrm{CP}}$, obtained by combining all sources in quadrature, are $2.1 \times 10^{-3}$ and $4.1 \times 10^{-3}$, respectively. 


\begin{tabular}{|l|c|c|}
\hline Effect & $A_{\Gamma}\left(10^{-3}\right)$ & $y_{\mathrm{CP}}\left(10^{-3}\right)$ \\
\hline Decay-time acceptance correction & 0.1 & 0.1 \\
Decay-time resolution & 0.1 & 0.1 \\
Minimum decay-time cut & 0.1 & 0.8 \\
Maximum decay-time cut & 0.2 & 0.2 \\
Combinatorial background & 1.3 & 0.8 \\
Secondary-like background & 1.6 & 3.9 \\
\hline Total & 2.1 & 4.1 \\
\hline
\end{tabular}

Table 1. Summary of systematic uncertainties.
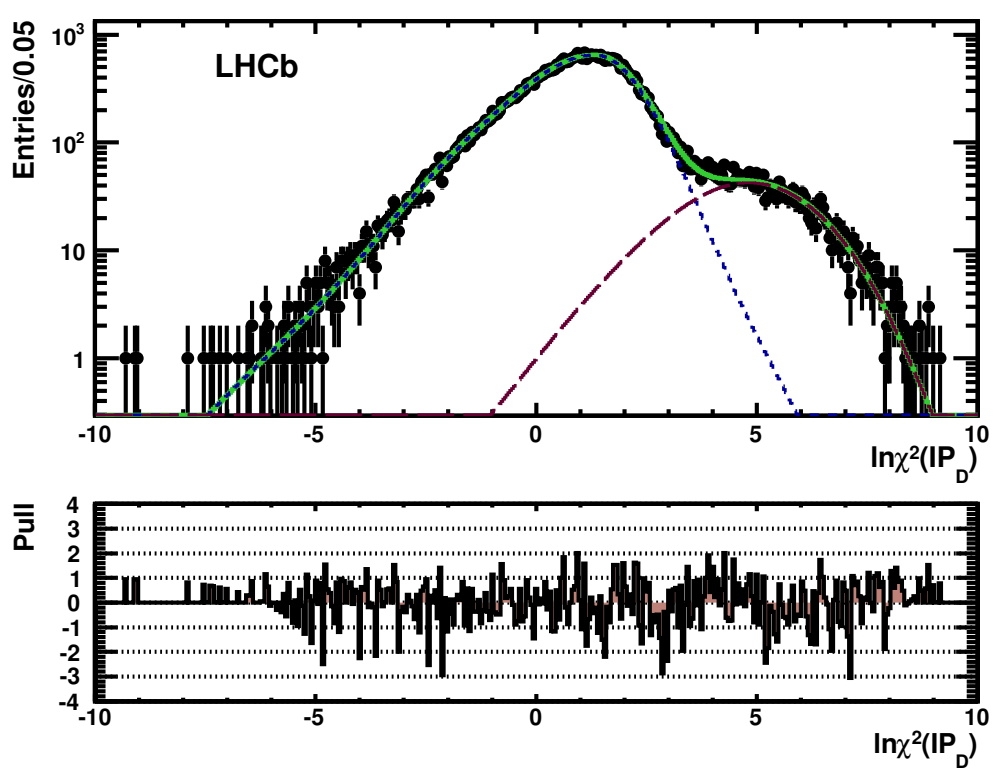

Figure 4. $\ln \chi^{2}\left(\mathrm{IP}_{D}\right)$ fit projection of $D^{0} \rightarrow K^{+} K^{-}$candidates in logarithmic scale. Shown are data (points), the total fit (green, solid), the prompt signal (blue, short-dashed), and the secondary signal (purple, long-dashed). The lower two plot shows the pull distribution which is defined as the difference of data and model divided by the uncertainty.

\section{Results and conclusion}

The measurement of $y_{\mathrm{CP}}$ is based on absolute lifetime measurements as described in section 4. It uses flavour-tagged events reconstructed in the decay chain $D^{*+} \rightarrow D^{0} \pi^{+}$, with $D^{0}$ and $\bar{D}^{0}$ decays fitted simultaneously per decay mode. The $\ln \chi^{2}\left(\operatorname{IP}_{D}\right)$ projection of the final fit is shown in figure 4 .

The result for the lifetime measured in $D^{0} \rightarrow K^{-} \pi^{+}$decays is $\tau\left(D^{0}\right)=410.2 \pm 0.9 \mathrm{fs}$ where the uncertainty is statistical only. The result for the lifetime is found to be in agreement with the current world average [19]. Combining with the $D^{0} \rightarrow K^{+} K^{-}$lifetime measurement, $\tau\left(D^{0}\right)=408.0 \pm 2.4_{\text {stat }}$ fs, this leads to the final result for $y_{\mathrm{CP}}$ of

$$
y_{\mathrm{CP}}=\left(5.5 \pm 6.3_{\text {stat }} \pm 4.1_{\text {syst }}\right) \times 10^{-3} .
$$



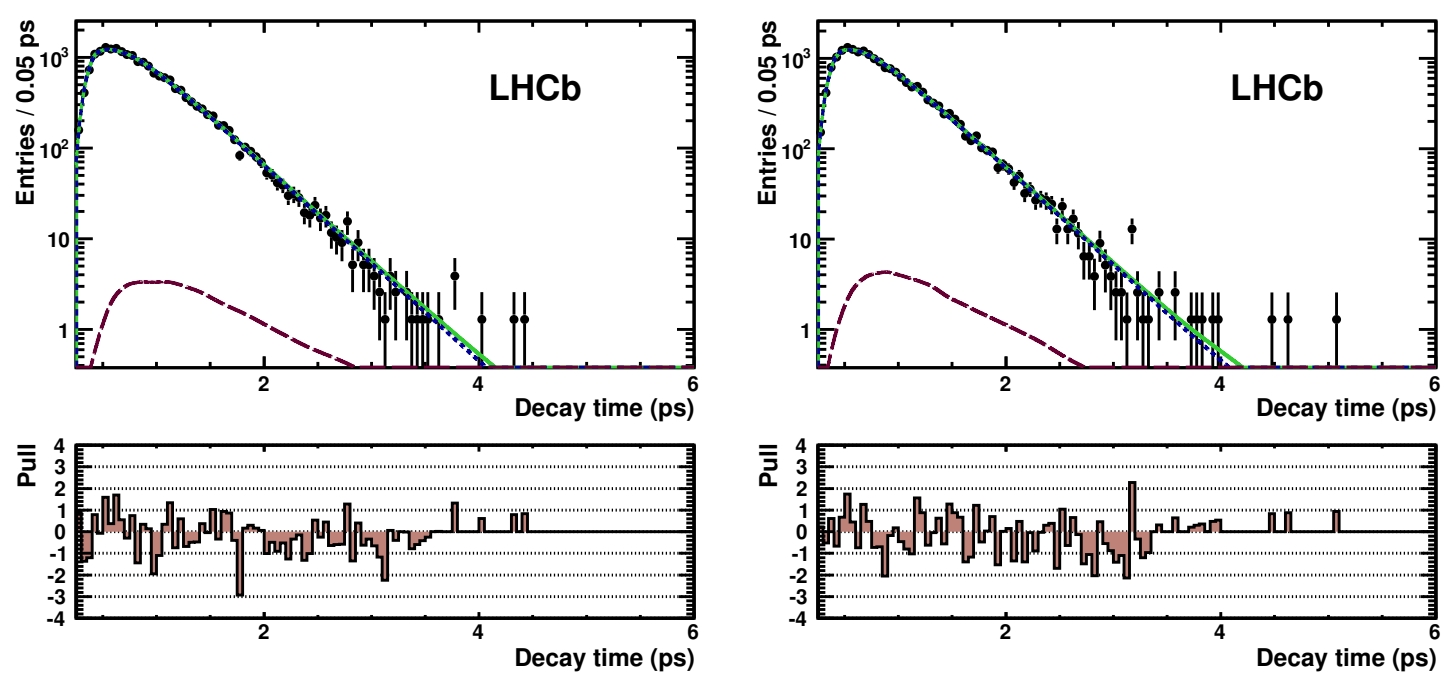

Figure 5. Proper-time fit projections of (left) $D^{0} \rightarrow K^{+} K^{-}$and (right) $\bar{D}^{0} \rightarrow K^{+} K^{-}$candidates after application of the $\ln \chi^{2}\left(\mathrm{IP}_{D}\right)<2$ cut. Shown are data (points), the total fit (green, solid), the prompt signal (blue, short-dashed), and the secondary signal (purple, long-dashed). The lower two plot shows the pull distribution which is defined as the difference of data and model divided by the uncertainty.

The measurement of $A_{\Gamma}$ is performed based on the same dataset and applying the same fitting method as used for the extraction of $y_{\mathrm{CP}}$. A control measurement is performed using decays to the Cabibbo favoured mode $D^{0} \rightarrow K^{-} \pi^{+}$by forming a lifetime asymmetry analogous to eq. (1.2). The measured flavour-tagged lifetimes are effective parameters since the fitted distributions also include mistagged events. For the control measurement using $D^{0} \rightarrow K^{-} \pi^{+}$decays this contamination is ignored as it is negligible due to the Cabibbo suppression of the mistagged decays. The result for the asymmetry is $A_{\Gamma}^{K \pi, \text { eff }}=$ $\left(-0.9 \pm 2.2_{\text {stat }}\right) \times 10^{-3}$ which is consistent with zero, according to expectations.

For the extraction of $A_{\Gamma}$, the mistagged decays are taken into account by expressing the measured effective lifetimes, $\tau^{\text {eff }}$, in terms of the flavour-tagged lifetimes, $\tau\left(D^{0}\right)$ and $\tau\left(\bar{D}^{0}\right)$, and the mistag rate, $\epsilon_{ \pm}$, where the sign is according to the sign of the tagging pion:

$$
\begin{aligned}
& \tau^{\mathrm{eff}}\left(D^{0}\right) \approx\left(1-\epsilon_{+}\right) \tau\left(D^{0}\right)+\epsilon_{+} \tau\left(\bar{D}^{0}\right) \\
& \tau^{\mathrm{eff}}\left(\bar{D}^{0}\right) \approx\left(1-\epsilon_{-}\right) \tau\left(\bar{D}^{0}\right)+\epsilon_{-} \tau\left(D^{0}\right) .
\end{aligned}
$$

The mistag rates are assumed to be independent of the final state and are extracted from the favoured $D^{0} \rightarrow K^{-} \pi^{+}$decays as half the fraction of the random slow pion background in the signal region of the $\Delta m$ distribution. They are found to be about $1.8 \%$. The systematic uncertainty due to this correction is negligible.

The projection of the decay-time fit to $D^{0}$ and $\bar{D}^{0}$ candidates in $D^{0} \rightarrow K^{+} K^{-}$decays is shown in figure 5. After applying the mistag correction, the resulting value of $A_{\Gamma}$ is

$$
A_{\Gamma}=\left(-5.9 \pm 5.9_{\text {stat }} \pm 2.1_{\text {syst }}\right) \times 10^{-3} \text {. }
$$


Both results on $y_{\mathrm{CP}}$ and $A_{\Gamma}$ are compatible with zero and in agreement with previous measurements $[2,8,9]$. Future updates are expected to lead to significant improvements in the sensitivity. The systematic uncertainty is expected to be reduced by an improved treatment of background events which will be possible for the data taken in 2011.

\section{Acknowledgments}

We express our gratitude to our colleagues in the CERN accelerator departments for the excellent performance of the LHC. We thank the technical and administrative staff at CERN and at the LHCb institutes, and acknowledge support from the National Agencies: CAPES, CNPq, FAPERJ and FINEP (Brazil); CERN; NSFC (China); CNRS/IN2P3 (France); BMBF, DFG, HGF and MPG (Germany); SFI (Ireland); INFN (Italy); FOM and NWO (The Netherlands); SCSR (Poland); ANCS (Romania); MinES of Russia and Rosatom (Russia); MICINN, XuntaGal and GENCAT (Spain); SNSF and SER (Switzerland); NAS Ukraine (Ukraine); STFC (United Kingdom); NSF (U.S.A.). We also acknowledge the support received from the ERC under FP7 and the Region Auvergne.

Open Access. This article is distributed under the terms of the Creative Commons Attribution License which permits any use, distribution and reproduction in any medium, provided the original author(s) and source are credited.

\section{References}

[1] BABAR collaboration, B. Aubert et al., Evidence for $D^{0} \bar{D}^{0}$ mixing, Phys. Rev. Lett. 98 (2007) 211802 [hep-ex/0703020] [INSPIRE].

[2] Belle collaboration, M. Staric et al., Evidence for $D^{0}-2$ mixing, Phys. Rev. Lett. 98 (2007) 211803 [hep-ex/0703036] [INSPIRE].

[3] BELLE collaboration, K. Abe et al., Measurement of $D^{0}-\bar{D}^{0}$ mixing parameters in $D^{0} \rightarrow K_{S}^{0} \pi^{+} \pi^{-}$decays, Phys. Rev. Lett. 99 (2007) 131803 [arXiv:0704.1000] [INSPIRE].

[4] LHCB collaboration, R. Aaij et al., Evidence for CP-violation in time-integrated $D^{0} \rightarrow h^{-} h^{+}$ decay rates, Phys. Rev. Lett. 108 (2012) 111602 [arXiv:1112.0938] [INSPIRE].

[5] Heavy Flavor Averaging Group collaboration, D. Asner et al., Averages of b-hadron, c-hadron and $\tau$-lepton properties, arXiv:1010.1589 [INSPIRE].

[6] S. Bergmann, Y. Grossman, Z. Ligeti, Y. Nir and A.A. Petrov, Lessons from 2 and 2 measurements of $D^{0}-\bar{D}^{0}$ mixing parameters, Phys. Lett. B 486 (2000) 418 [hep-ph/0005181] [INSPIRE].

[7] M. Gersabeck, M. Alexander, S. Borghi, V. Gligorov and C. Parkes, On the interplay of direct and indirect CP-violation in the charm sector, J. Phys. G 39 (2012) 045005 [arXiv:1111.6515] [INSPIRE].

[8] BABAR collaboration, B. Aubert et al., Measurement of $D^{0}-\bar{D}^{0}$ Mixing using the Ratio of Lifetimes for the Decays D0 -i K- pit and K+K-, Phys. Rev. D 80 (2009) 071103 [arXiv: 0908.0761] [INSPIRE]. 
[9] BABAR collaboration, B. Aubert et al., Measurement of $D^{0}-\bar{D}^{0}$ mixing using the ratio of lifetimes for the decays $D^{0} \rightarrow K^{-} \pi^{+}, K^{-} K^{+}$and $\pi^{-} \pi^{+}$, Phys. Rev. D 78 (2008) 011105 [arXiv:0712.2249] [INSPIRE].

[10] LHCB collaboration, J. Alves, A. Augusto et al., The LHCb Detector at the LHC, 2008 JINST 3 S08005 [INSPIRE].

[11] R. Bailey, E. Belau, T. Bohringer, M. Bosman, V. Chabaud, et al., Measurement of the lifetime of charged and neutral $D$ mesons with high resolution silicon strip detectors, Z. Phys. C 28 (1985) 357 [INSPIRE].

[12] DELPHI collaboration, W. Adam et al., Lifetimes of charged and neutral B hadrons using event topology, Z. Phys. C 68 (1995) 363 [INSPIRE].

[13] J. Rademacker, Reduction of statistical power per event due to upper lifetime cuts in lifetime measurements, Nucl. Instrum. Meth. A 570 (2007) 525 [hep-ex/0502042] [INSPIRE].

[14] CDF collaboration, T. Aaltonen et al., Measurement of the $B^{-}$lifetime using a simulation free approach for trigger bias correction, Phys. Rev. D 83 (2011) 032008 [arXiv:1004.4855] [INSPIRE].

[15] V.V. Gligorov, Measurement of the CKM angle gamma and B meson lifetimes at the LHCb detector, Ph.D. Thesis, St. John's college, Ofxord U.K. (2008) [CERN-THESIS-2008-044] [INSPIRE].

[16] M. Gersabeck, Alignment of the LHCb vertex locator and lifetime measurements of two-body hadronic final states, Ph.D. Thesis, Galsgow University, Glasgow U.K. (2009) [CERN-THESIS-2009-118] [INSPIRE].

[17] LHCB collaboration, R. Aaij et al., Measurement of the effective $B_{s}^{0} \rightarrow K^{+} K^{-}$lifetime, Phys. Lett. B 707 (2012) 349 [arXiv:1111.0521] [INSPIRE].

[18] LHCb Collaboration, R. Aaij et al., Measurement of the CP-violating phase $\phi_{s}$ in the decay $B_{s}^{0} \rightarrow J / \psi \phi$, Phys. Rev. Lett. 108 (2012) 101803 [arXiv:1112.3183] [InSPIRE].

[19] Particle Data Group collaboration, K. Nakamura et al., Review of particle physics, J. Phys. G 37 (2010) 075021 [inSPIRE]. 


\section{The LHCb collaboration}

R. Aaij ${ }^{38}$, C. Abellan Beteta ${ }^{33, n}$, B. Adeva ${ }^{34}$, M. Adinolfi ${ }^{43}$, C. Adrover ${ }^{6}$, A. Affolder ${ }^{49}$, Z. Ajaltouni ${ }^{5}$, J. Albrecht ${ }^{35}$, F. Alessio ${ }^{35}$, M. Alexander ${ }^{48}$, S. Ali ${ }^{38}$, G. Alkhazov ${ }^{27}$, P. Alvarez Cartelle ${ }^{34}$, A.A. Alves Jr22, S. Amato ${ }^{2}$, Y. Amhis ${ }^{36}$, J. Anderson ${ }^{37}$, R.B. Appleby ${ }^{51}$, O. Aquines Gutierrez ${ }^{10}$, F. Archilli ${ }^{18,35}$, A. Artamonov ${ }^{32}$, M. Artuso ${ }^{53,35}$, E. Aslanides ${ }^{6}$, G. Auriemma ${ }^{22, m}$, S. Bachmann ${ }^{11}$, J.J. Back ${ }^{45}$, V. Balagura ${ }^{28,35}$, W. Baldini ${ }^{16}$, R.J. Barlow ${ }^{51}$, C. Barschel ${ }^{35}$, S. Barsuk ${ }^{7}$, W. Barter ${ }^{44}$, A. Bates ${ }^{48}$, C. Bauer ${ }^{10}$, Th. Bauer ${ }^{38}$, A. Bay ${ }^{36}$, I. Bediaga ${ }^{1}$, S. Belogurov ${ }^{28}$, K. Belous ${ }^{32}$, I. Belyaev ${ }^{28}$, E. Ben-Haim ${ }^{8}$, M. Benayoun ${ }^{8}$, G. Bencivenni ${ }^{18}$, S. Benson ${ }^{47}$, J. Benton ${ }^{43}$, R. Bernet ${ }^{37}$, M.-O. Bettler ${ }^{17}$, M. van Beuzekom ${ }^{38}$, A. Bien ${ }^{11}$, S. Bifani ${ }^{12}$, T. Bird $^{51}$, A. Bizzeti ${ }^{17, h}$, P.M. Bjørnstad ${ }^{51}$, T. Blake ${ }^{35}$, F. Blanc ${ }^{36}$, C. Blanks ${ }^{50}$, J. Blouw ${ }^{11}$, S. Blusk ${ }^{53}$, A. Bobrov ${ }^{31}$, V. Bocci ${ }^{22}$, A. Bondar ${ }^{31}$, N. Bondar ${ }^{27}$, W. Bonivento ${ }^{15}$, S. Borghi ${ }^{48,51}$, A. Borgia ${ }^{53}$, T.J.V. Bowcock ${ }^{49}$, C. Bozzi ${ }^{16}$, T. Brambach ${ }^{9}$, J. van den Brand ${ }^{39}$,

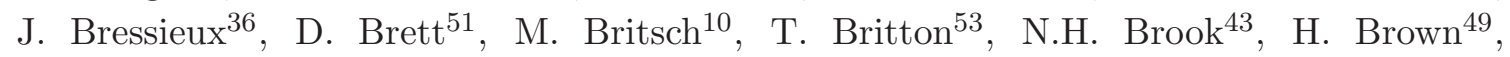
K. de Bruyn ${ }^{38}$, A. Büchler-Germann ${ }^{37}$, I. Burducea ${ }^{26}$, A. Bursche ${ }^{37}$, J. Buytaert ${ }^{35}$, S. Cadeddu ${ }^{15}$, O. Callot ${ }^{7}$, M. Calvi ${ }^{20, j}$, M. Calvo Gomez ${ }^{33, n}$, A. Camboni ${ }^{33}$, P. Campana ${ }^{18,35}$, A. Carbone ${ }^{14}$, G. Carboni ${ }^{21, k}$, R. Cardinale ${ }^{19, i, 35}$, A. Cardini ${ }^{15}$, L. Carson ${ }^{50}$, K. Carvalho Akiba ${ }^{2}$, G. Casse ${ }^{49}$, M. Cattaneo ${ }^{35}$, Ch. Cauet ${ }^{9}$, M. Charles ${ }^{52}$, Ph. Charpentier ${ }^{35}$, N. Chiapolini ${ }^{37}$, K. $\mathrm{Ciba}^{35}$, X. Cid Vidal ${ }^{34}$, G. Ciezarek $^{50}$, P.E.L. Clarke ${ }^{47,35}$, M. Clemencic ${ }^{35}$, H.V. Cliff ${ }^{44}$, J. Closier ${ }^{35}$, C. Coca ${ }^{26}$, V. Coco $^{38}$, J. Cogan ${ }^{6}$, P. Collins ${ }^{35}$, A. Comerma-Montells ${ }^{33}$, A. Contu ${ }^{52}$, A. Cook ${ }^{43}$, M. Coombes ${ }^{43}$,

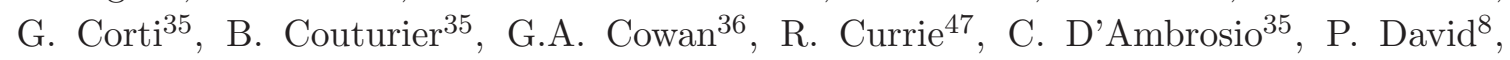
P.N.Y. David ${ }^{38}$, I. De Bonis ${ }^{4}$, S. De Capua ${ }^{21, k}$, M. De Cian ${ }^{37}$, J.M. De Miranda ${ }^{1}$, L. De Paula ${ }^{2}$, P. De Simone ${ }^{18}$, D. Decamp ${ }^{4}$, M. Deckenhoff ${ }^{9}$, H. Degaudenzi ${ }^{36,35}$, L. Del Buono ${ }^{8}$, C. Deplano ${ }^{15}$, D. Derkach ${ }^{14,35}$, O. Deschamps ${ }^{5}$, F. Dettori ${ }^{39}$, J. Dickens ${ }^{44}$, H. Dijkstra ${ }^{35}$, P. Diniz Batista ${ }^{1}$, F. Domingo Bonal ${ }^{33, n}$, S. Donleavy ${ }^{49}$, F. Dordei ${ }^{11}$, A. Dosil Suárez ${ }^{34}$, D. Dossett ${ }^{45}$, A. Dovbnya ${ }^{40}$, F. Dupertuis ${ }^{36}$, R. Dzhelyadin ${ }^{32}$, A. Dziurda ${ }^{23}$, S. Easo ${ }^{46}$, U. Egede ${ }^{50}$, V. Egorychev ${ }^{28}$, S. Eidelman ${ }^{31}$, D. van Eijk ${ }^{38}$, F. Eisele ${ }^{11}$, S. Eisenhardt ${ }^{47}$, R. Ekelhof ${ }^{9}$, L. Eklund ${ }^{48}$, Ch. Elsasser ${ }^{37}$, D. Elsby ${ }^{42}$, D. Esperante Pereira ${ }^{34}$, A. Falabella ${ }^{16, e, 14}$, C. Färber ${ }^{11}$, G. Fardell ${ }^{47}$, C. Farinelli ${ }^{38}$, S. Farry ${ }^{12}$, V. Fave ${ }^{36}$, V. Fernandez Albor ${ }^{34}$, M. Ferro-Luzzi ${ }^{35}$, S. Filippov ${ }^{30}$, C. Fitzpatrick ${ }^{47}$, M. Fontana ${ }^{10}$, F. Fontanelli ${ }^{19, i}$, R. Forty ${ }^{35}$, O. Francisco ${ }^{2}$, M. Frank ${ }^{35}$, C. Frei ${ }^{35}$, M. Frosini ${ }^{17, f}$, S. Furcas ${ }^{20}$, A. Gallas Torreira ${ }^{34}$, D. Galli ${ }^{14, c}$, M. Gandelman ${ }^{2}$, P. Gandini ${ }^{52}$, Y. $\mathrm{Gao}^{3}$, J-C. Garnier ${ }^{35}$, J. Garofoli ${ }^{53}$, J. Garra Tico ${ }^{44}$, L. Garrido ${ }^{33}$, D. Gascon ${ }^{33}$, C. $G a s p a r{ }^{35}$, R. Gauld ${ }^{52}$, N. Gauvin ${ }^{36}$, M. Gersabeck ${ }^{35}$, T. Gershon ${ }^{45,35}$, Ph. Ghez ${ }^{4}$, V. Gibson ${ }^{44}$, V.V. Gligorov ${ }^{35}$, C. Göbel ${ }^{54}$, D. Golubkov ${ }^{28}$, A. Golutvin ${ }^{50,28,35}$, A. Gomes ${ }^{2}$, H. Gordon ${ }^{52}$, M. Grabalosa Gándara ${ }^{33}$, R. Graciani Diaz ${ }^{33}$, L.A. Granado Cardoso ${ }^{35}$, E. Graugés ${ }^{33}$, G. Graziani ${ }^{17}$, A. Grecu ${ }^{26}$, E. Greening ${ }^{52}$, S. Gregson ${ }^{44}$, B. Gui ${ }^{53}$, E. Gushchin ${ }^{30}$, Yu. Guz ${ }^{32}$, T. Gys ${ }^{35}$, C. Hadjivasiliou ${ }^{53}$, G. Haefeli ${ }^{36}$, C. Haen ${ }^{35}$, S.C. Haines ${ }^{44}$, T. Hampson ${ }^{43}$, S. Hansmann-Menzemer ${ }^{11}$, R. Harji ${ }^{50}$, N. Harnew ${ }^{52}$, J. Harrison ${ }^{51}$, P.F. Harrison ${ }^{45}$, T. Hartmann ${ }^{55}$, J. He ${ }^{7}$, V. Heijne ${ }^{38}$, K. Hennessy ${ }^{49}$, P. Henrard ${ }^{5}$, J.A. Hernando Morata ${ }^{34}$, E. van Herwijnen ${ }^{35}$, E. Hicks ${ }^{49}$, K. Holubyev ${ }^{11}$, 


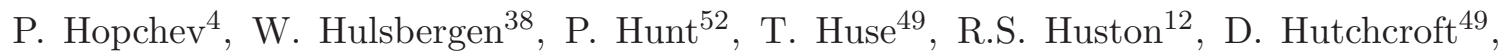
D. Hynds ${ }^{48}$, V. Iakovenko ${ }^{41}$, P. Ilten ${ }^{12}$, J. Imong ${ }^{43}$, R. Jacobsson ${ }^{35}$, A. Jaeger ${ }^{11}$, M. Jahjah Hussein ${ }^{5}$, E. Jans ${ }^{38}$, F. Jansen ${ }^{38}$, P. Jaton ${ }^{36}$, B. Jean-Marie ${ }^{7}$, F. Jing ${ }^{3}$, M. John ${ }^{52}$, D. Johnson ${ }^{52}$, C.R. Jones ${ }^{44}$, B. Jost ${ }^{35}$, M. Kaballo ${ }^{9}$, S. Kandybei ${ }^{40}$, M. Karacson ${ }^{35}$, T.M. Karbach ${ }^{9}$, J. Keaveney ${ }^{12}$, I.R. Kenyon ${ }^{42}$, U. Kerzel ${ }^{35}$, T. Ketel ${ }^{39}$, A. Keune ${ }^{36}$, B. Khanji ${ }^{6}$, Y.M. Kim ${ }^{47}$, M. Knecht ${ }^{36}$, R.F. Koopman ${ }^{39}$, P. Koppenburg ${ }^{38}$, M. Korolev ${ }^{29}$, A. Kozlinskiy ${ }^{38}$, L. Kravchuk ${ }^{30}$, K. Kreplin ${ }^{11}$, M. Kreps ${ }^{45}$, G. Krocker ${ }^{11}$, P. Krokovny ${ }^{11}$, F. Kruse ${ }^{9}$, K. Kruzelecki ${ }^{35}$, M. Kucharczyk ${ }^{20,23,35, j}$, V. Kudryavtsev ${ }^{31}$, T. Kvaratskheliya ${ }^{28,35}$, V.N. La Thi ${ }^{36}$, D. Lacarrere ${ }^{35}$, G. Lafferty ${ }^{51}$, A. Lai ${ }^{15}$, D. Lambert ${ }^{47}$, R.W. Lambert ${ }^{39}$, E. Lanciotti ${ }^{35}$, G. Lanfranchi ${ }^{18}$, C. Langenbruch ${ }^{11}$,

T. Latham ${ }^{45}$, C. Lazzeroni ${ }^{42}$, R. Le Gac ${ }^{6}$, J. van Leerdam ${ }^{38}$, J.-P. Lees ${ }^{4}$, R. Lefèvre ${ }^{5}$, A. Leflat ${ }^{29,35}$, J. Lefrançois ${ }^{7}$, O. Leroy ${ }^{6}$, T. Lesiak ${ }^{23}$, L. Li ${ }^{3}$, L. Li Gioi ${ }^{5}$, M. Lieng ${ }^{9}$, M. Liles ${ }^{49}$, R. Lindner ${ }^{35}$, C. Linn ${ }^{11}$, B. Liu ${ }^{3}$, G. Liu ${ }^{35}$, J. von Loeben ${ }^{20}$, J.H. Lopes ${ }^{2}$, E. Lopez Asamar ${ }^{33}$, N. Lopez-March ${ }^{36}$, H. Lu ${ }^{3}$, J. Luisier ${ }^{36}$, A. Mac Raighne ${ }^{48}$, F. Machefert ${ }^{7}$, I.V. Machikhiliyan ${ }^{4,28}$, F. Maciuc ${ }^{10}$, O. Maev ${ }^{27,35}$, J. Magnin ${ }^{1}$, S. Malde ${ }^{52}$, R.M.D. Mamunur ${ }^{35}$, G. Manca ${ }^{15, d}$, G. Mancinelli ${ }^{6}$, N. Mangiafave ${ }^{44}$, U. Marconi ${ }^{14}$, R. Märki ${ }^{36}$, J. Markss ${ }^{11}$, G. Martellotti' ${ }^{22}$, A. Martens ${ }^{8}$, L. Martin ${ }^{52}$, A. Martín Sánchez ${ }^{7}$, M. Martinelli ${ }^{38}$, D. Martinez Santos ${ }^{35}$, A. Massafferri ${ }^{1}$, Z. Mathe ${ }^{12}$, C. Matteuzzi ${ }^{20}$, M. Matveev $^{27}$, E. Maurice ${ }^{6}$, B. Maynard ${ }^{53}$, A. Mazurov ${ }^{16,30,35}$, G. McGregor ${ }^{51}$, R. McNulty ${ }^{12}$, M. Meissner ${ }^{11}$, M. Merk ${ }^{38}$, J. Merkel ${ }^{9}$, S. Miglioranzi ${ }^{35}$, D.A. Milanes ${ }^{13}$, M.-N. Minard ${ }^{4}$, J. Molina Rodriguez ${ }^{54}$, S. Monteil ${ }^{5}$, D. Moran ${ }^{12}$, P. Morawski ${ }^{23}$, R. Mountain ${ }^{53}$, I. Mous ${ }^{38}$, F. Muheim ${ }^{47}$, K. Müller ${ }^{37}$, R. Muresan ${ }^{26}$, B. Muryn ${ }^{24}$, B. Muster ${ }^{36}$, J. Mylroie-Smith ${ }^{49}$, P. Naik ${ }^{43}$, T. Nakada ${ }^{36}$, R. Nandakumar ${ }^{46}$, I. Nasteva ${ }^{1}$, M. Needham ${ }^{47}$, N. Neufeld ${ }^{35}$, A.D. Nguyen ${ }^{36}$, C. Nguyen-Mau ${ }^{36, o}, \mathrm{M} . \mathrm{Nicol}^{7}, \mathrm{~V} . \mathrm{Niess}^{5}$, N. Nikitin ${ }^{29}$, A. Nomerotski ${ }^{52,35}$, A. Novoselov ${ }^{32}$, A. Oblakowska-Mucha ${ }^{24}$, V. Obraztsov ${ }^{32}$, S. Oggero $^{38}$, S. Ogilvy ${ }^{48}$, O. Okhrimenko ${ }^{41}$, R. Oldeman ${ }^{15, d, 35}$, M. Orlandea ${ }^{26}$, J.M. Otalora Goicochea ${ }^{2}$, P. Owen ${ }^{50}$, K. Pal ${ }^{53}$, J. Palacios ${ }^{37}$, A. Palano ${ }^{13, b}$, M. Palutan ${ }^{18}$, J. Panman ${ }^{35}$, A. Papanestis ${ }^{46}$, M. Pappagallo ${ }^{48}$, C. Parkes ${ }^{51}$, C.J. Parkinson ${ }^{50}$, G. Passaleva ${ }^{17}$, G.D. Patel ${ }^{49}$, M. Patel ${ }^{50}$, S.K. Paterson ${ }^{50}$, G.N. Patrick ${ }^{46}$, C. Patrignani ${ }^{19, i}$, C. Pavel-Nicorescu ${ }^{26}$, A. Pazos Alvarez ${ }^{34}$, A. Pellegrino ${ }^{38}$, G. Penso ${ }^{22, l}$, M. Pepe Altarelli ${ }^{35}$, S. Perazzini ${ }^{14, c}$, D.L. Perego ${ }^{20, j}$, E. Perez Trigo ${ }^{34}$, A. Pérez-Calero Yzquierdo ${ }^{33}$, P. Perret $^{5}$, M. Perrin-Terrin ${ }^{6}$, G. Pessina ${ }^{20}$, A. Petrolini ${ }^{19, i}$, A. Phan ${ }^{53}$, E. Picatoste Olloqui ${ }^{33}$, B. Pie Valls ${ }^{33}$, B. Pietrzyk ${ }^{4}$, T. Pilař ${ }^{45}$, D. Pinci ${ }^{22}$, R. Plackett ${ }^{48}$, S. Playfer ${ }^{47}$, M. Plo Casasus ${ }^{34}$, G. Polok ${ }^{23}$, A. Poluektov ${ }^{45,31}$, E. Polycarpo ${ }^{2}$, D. Popov ${ }^{10}$, B. Popovici ${ }^{26}$, C. Potterat ${ }^{33}$, A. Powell ${ }^{52}$, J. Prisciandaro ${ }^{36}$, V. Pugatch ${ }^{41}$, A. Puig Navarro ${ }^{33}$, W. $\operatorname{Qian}^{53}$, J.H. Rademacker ${ }^{43}$, B. Rakotomiaramanana ${ }^{36}$, M.S. Rangel ${ }^{2}$, I. Raniuk ${ }^{40}$, G. $R^{2}{ }^{39}$, S. Redford ${ }^{52}$, M.M. Reid ${ }^{45}$, A.C. dos Reis ${ }^{1}$, S. Ricciardi ${ }^{46}$, A. Richards ${ }^{50}$, K. Rinnert ${ }^{49}$, D.A. Roa Romero ${ }^{5}$, P. Robbe ${ }^{7}$, E. Rodrigues ${ }^{48,51}$, F. Rodrigues ${ }^{2}$, P. Rodriguez Perez ${ }^{34}$, G.J. Rogers ${ }^{44}$, S. Roiser ${ }^{35}$, V. Romanovsky ${ }^{32}$, M. Rosello ${ }^{33, n}$, J. Rouvinet ${ }^{36}, \quad$ T. $\operatorname{Ruf}^{35}, \quad$ H. Ruiz ${ }^{33}$, G. Sabatino ${ }^{21, k}$, J.J. Saborido Silva ${ }^{34}$, N. Sagidova ${ }^{27}$, P. Sail ${ }^{48}$, B. Saitta ${ }^{15, d}$, C. Salzmann ${ }^{37}$, M. Sannino ${ }^{19, i}$, R. Santacesaria ${ }^{22}$, C. Santamarina Rios ${ }^{34}$, R. Santinelli ${ }^{35}$, E. Santovetti ${ }^{21, k}$, M. Sapunov ${ }^{6}$, A. $\operatorname{Sarti}^{18, l}$, C. Satriano ${ }^{22, m}$, A. Satta ${ }^{21}$, M. Savrie ${ }^{16, e}$, D. Savrina ${ }^{28}$, P. Schaack ${ }^{50}$, M. Schiller ${ }^{39}$, 
H. Schindler ${ }^{35}$, S. Schleich ${ }^{9}$, M. Schlupp ${ }^{9}$, M. Schmelling ${ }^{10}$, B. Schmidt ${ }^{35}$, O. Schneider ${ }^{36}$, A. Schopper ${ }^{35}$, M.-H. Schune ${ }^{7}$, R. Schwemmer ${ }^{35}$, B. Sciascia ${ }^{18}$, A. Sciubba ${ }^{18, l}$, M. Seco ${ }^{34}$, A. Semennikov ${ }^{28}$, K. Senderowska ${ }^{24}$, I. Sepp ${ }^{50}$, N. Serra ${ }^{37}$, J. Serrano ${ }^{6}$, P. Seyfert ${ }^{11}$, M. Shapkin ${ }^{32}$, I. Shapoval ${ }^{40,35}$, P. Shatalov ${ }^{28}$, Y. Shcheglov ${ }^{27}$, T. Shears ${ }^{49}$, L. Shekhtman ${ }^{31}$, O. Shevchenko ${ }^{40}$, V. Shevchenko ${ }^{28}$, A. Shires ${ }^{50}$, R. Silva Coutinho ${ }^{45}$, T. Skwarnicki ${ }^{53}$, N.A. Smith ${ }^{49}$, E. Smith ${ }^{52,46}$, K. Sobczak ${ }^{5}$, F.J.P. Soler ${ }^{48}$, A. Solomin ${ }^{43}$, F. Soomro ${ }^{18,35}$, B. Souza De Paula $^{2}$, B. Spaan $^{9}$, A. Sparkes ${ }^{47}$, P. Spradlin ${ }^{48}$, F. Stagni ${ }^{35}$, S. Stahl ${ }^{11}$, O. Steinkamp ${ }^{37}$, S. Stoica ${ }^{26}$, S. Stone ${ }^{53,35}$, B. Storaci ${ }^{38}$, M. Straticiuc ${ }^{26}$, U. Straumann ${ }^{37}$, V.K. Subbiah ${ }^{35}$, S. Swientek ${ }^{9}$, M. Szczekowski ${ }^{25}$, P. Szczypka ${ }^{36}$, T. Szumlak ${ }^{24}$, S. T'Jampens ${ }^{4}$, E. Teodorescu ${ }^{26}$, F. Teubert ${ }^{35}$, C. Thomas ${ }^{52}$, E. Thomas ${ }^{35}$, J. van Tilburg ${ }^{11}$, V. Tisserand ${ }^{4}$, M. Tobin ${ }^{37}$, S. Topp-Joergensen ${ }^{52}$, N. Torr ${ }^{52}$, E. Tournefier ${ }^{4,50}$, S. Tourneur ${ }^{36}$, M.T. Tran ${ }^{36}$, A. Tsaregorodtsev 6 , N. Tuning ${ }^{38}$, M. Ubeda Garcia ${ }^{35}$, A. Ukleja ${ }^{25}$, U. Uwer ${ }^{11}$, V. Vagnoni ${ }^{14}$, G. Valenti ${ }^{14}$, R. Vazquez Gomez ${ }^{33}$, P. Vazquez Regueiro ${ }^{34}$, S. Vecchi ${ }^{16}$, J.J. Velthuis ${ }^{43}$, M. Veltri ${ }^{17, g}$, B. Viaud ${ }^{7}$, I. Videau ${ }^{7}$, D. Vieira ${ }^{2}$, X. Vilasis-Cardona ${ }^{33, n}$, J. Visniakov ${ }^{34}$, A. Vollhardt ${ }^{37}$, D. Volyanskyy ${ }^{10}$, D. Voong ${ }^{43}$, A. Vorobyev ${ }^{27}, \mathrm{H} . \mathrm{Voss}^{10}$, R. Waldi ${ }^{55}$, S. Wandernoth ${ }^{11}$, J. Wang ${ }^{53}$, D.R. Ward ${ }^{44}$, N.K. Watson ${ }^{42}$, A.D. Webber ${ }^{51}$, D. Websdale ${ }^{50}$, M. Whitehead ${ }^{45}$, D. Wiedner ${ }^{11}$, L. Wiggers ${ }^{38}$, G. Wilkinson ${ }^{52}$, M.P. Williams ${ }^{45,46}$, M. Williams ${ }^{50}$, F.F. Wilson ${ }^{46}$, J. Wishahi ${ }^{9}$, M. Witek ${ }^{23}$, W. Witzeling ${ }^{35}$, S.A. Wotton ${ }^{44}$, K. Wyllie ${ }^{35}$, Y. Xie ${ }^{47}$, F. Xing ${ }^{52}$, Z. Xing ${ }^{53}$, Z. Yang ${ }^{3}$, R. Young ${ }^{47}$, O. Yushchenko ${ }^{32}$, M. Zangoli ${ }^{14}$, M. Zavertyaev ${ }^{10, a}$, F. Zhang ${ }^{3}$, L. Zhang ${ }^{53}$, W.C. Zhang ${ }^{12}$, Y. Zhang ${ }^{3}$, A. Zhelezov ${ }^{11}$, L. Zhong ${ }^{3}$, A. Zvyagin ${ }^{35}$.

${ }^{1}$ Centro Brasileiro de Pesquisas Físicas (CBPF), Rio de Janeiro, Brazil

${ }^{2}$ Universidade Federal do Rio de Janeiro (UFRJ), Rio de Janeiro, Brazil

${ }^{3}$ Center for High Energy Physics, Tsinghua University, Beijing, China

${ }^{4}$ LAPP, Université de Savoie, CNRS/IN2P3, Annecy-Le-Vieux, France

${ }^{5}$ Clermont Université, Université Blaise Pascal, CNRS/IN2P3, LPC, Clermont-Ferrand, France

${ }^{6}$ CPPM, Aix-Marseille Université, CNRS/IN2P3, Marseille, France

${ }^{7}$ LAL, Université Paris-Sud, CNRS/IN2P3, Orsay, France

${ }^{8}$ LPNHE, Université Pierre et Marie Curie, Université Paris Diderot, CNRS/IN2P3, Paris, France

${ }^{9}$ Fakultät Physik, Technische Universität Dortmund, Dortmund, Germany

${ }^{10}$ Max-Planck-Institut für Kernphysik (MPIK), Heidelberg, Germany

${ }^{11}$ Physikalisches Institut, Ruprecht-Karls-Universität Heidelberg, Heidelberg, Germany

${ }^{12}$ School of Physics, University College Dublin, Dublin, Ireland

${ }^{13}$ Sezione INFN di Bari, Bari, Italy

${ }^{14}$ Sezione INFN di Bologna, Bologna, Italy

${ }^{15}$ Sezione INFN di Cagliari, Cagliari, Italy

${ }^{16}$ Sezione INFN di Ferrara, Ferrara, Italy

${ }^{17}$ Sezione INFN di Firenze, Firenze, Italy

${ }^{18}$ Laboratori Nazionali dell'INFN di Frascati, Frascati, Italy

${ }^{19}$ Sezione INFN di Genova, Genova, Italy

${ }^{20}$ Sezione INFN di Milano Bicocca, Milano, Italy

${ }^{21}$ Sezione INFN di Roma Tor Vergata, Roma, Italy 
${ }^{22}$ Sezione INFN di Roma La Sapienza, Roma, Italy

${ }^{23}$ Henryk Niewodniczanski Institute of Nuclear Physics Polish Academy of Sciences, Kraków, Poland

${ }^{24}$ AGH University of Science and Technology, Kraków, Poland

${ }^{25}$ Soltan Institute for Nuclear Studies, Warsaw, Poland

${ }^{26}$ Horia Hulubei National Institute of Physics and Nuclear Engineering, Bucharest-Magurele, Romania

${ }^{27}$ Petersburg Nuclear Physics Institute (PNPI), Gatchina, Russia

${ }^{28}$ Institute of Theoretical and Experimental Physics (ITEP), Moscow, Russia

${ }^{29}$ Institute of Nuclear Physics, Moscow State University (SINP MSU), Moscow, Russia

${ }^{30}$ Institute for Nuclear Research of the Russian Academy of Sciences (INR RAN), Moscow, Russia

${ }^{31}$ Budker Institute of Nuclear Physics (SB RAS) and Novosibirsk State University, Novosibirsk, Russia

${ }^{32}$ Institute for High Energy Physics (IHEP), Protvino, Russia

${ }^{33}$ Universitat de Barcelona, Barcelona, Spain

${ }^{34}$ Universidad de Santiago de Compostela, Santiago de Compostela, Spain

${ }^{35}$ European Organization for Nuclear Research (CERN), Geneva, Switzerland

${ }^{36}$ Ecole Polytechnique Fédérale de Lausanne (EPFL), Lausanne, Switzerland

${ }^{37}$ Physik-Institut, Universität Zürich, Zürich, Switzerland

${ }^{38}$ Nikhef National Institute for Subatomic Physics, Amsterdam, The Netherlands

${ }^{39}$ Nikhef National Institute for Subatomic Physics and Vrije Universiteit, Amsterdam, The Netherlands

${ }^{40}$ NSC Kharkiv Institute of Physics and Technology (NSC KIPT), Kharkiv, Ukraine

${ }^{41}$ Institute for Nuclear Research of the National Academy of Sciences (KINR), Kyiv, Ukraine

${ }^{42}$ University of Birmingham, Birmingham, United Kingdom

${ }^{43}$ H.H. Wills Physics Laboratory, University of Bristol, Bristol, United Kingdom

${ }^{44}$ Cavendish Laboratory, University of Cambridge, Cambridge, United Kingdom

${ }^{45}$ Department of Physics, University of Warwick, Coventry, United Kingdom

${ }^{46}$ STFC Rutherford Appleton Laboratory, Didcot, United Kingdom

${ }^{47}$ School of Physics and Astronomy, University of Edinburgh, Edinburgh, United Kingdom

${ }^{48}$ School of Physics and Astronomy, University of Glasgow, Glasgow, United Kingdom

${ }^{49}$ Oliver Lodge Laboratory, University of Liverpool, Liverpool, United Kingdom

${ }^{50}$ Imperial College London, London, United Kingdom

${ }^{51}$ School of Physics and Astronomy, University of Manchester, Manchester, United Kingdom

${ }^{52}$ Department of Physics, University of Oxford, Oxford, United Kingdom

${ }^{53}$ Syracuse University, Syracuse, NY, United States

${ }^{54}$ Pontifícia Universidade Católica do Rio de Janeiro (PUC-Rio), Rio de Janeiro, Brazil, associated to ${ }^{2}$

${ }^{55}$ Physikalisches Institut, Universität Rostock, Rostock, Germany, associated to ${ }^{11}$

${ }^{a}$ P.N. Lebedev Physical Institute, Russian Academy of Science (LPI RAS), Moscow, Russia

${ }^{b}$ Università di Bari, Bari, Italy

${ }^{c}$ Università di Bologna, Bologna, Italy

${ }^{d}$ Università di Cagliari, Cagliari, Italy

e Università di Ferrara, Ferrara, Italy

${ }^{f}$ Università di Firenze, Firenze, Italy

${ }^{g}$ Università di Urbino, Urbino, Italy

${ }^{h}$ Università di Modena e Reggio Emilia, Modena, Italy

${ }^{i}$ Università di Genova, Genova, Italy 
${ }^{j}$ Università di Milano Bicocca, Milano, Italy

${ }^{k}$ Università di Roma Tor Vergata, Roma, Italy

${ }^{l}$ Università di Roma La Sapienza, Roma, Italy

${ }^{m}$ Università della Basilicata, Potenza, Italy

${ }^{n}$ LIFAELS, La Salle, Universitat Ramon Llull, Barcelona, Spain

${ }^{\circ}$ Hanoi University of Science, Hanoi, Viet Nam 